\title{
EXISTENCE OF SOLUTIONS OF OSEEN TYPE INTEGRAL EQUATIONS
}

\author{
J.A. BELWARD
}

\begin{abstract}
Integral equations of Oseen type are first kind Fredholm equations whose kernels have a logarithmic singularity. They arise in exterior boundary value problems in fluid flow and heat transfer. Subject to the assumption of uniqueness of solutions of the parent exterior boundary value problem, solutions of the Oseen type integral equations are shown to exist.
\end{abstract}

\section{Introduction}

Integral equation methods are being used with increasing frequency to solve problems in applied mathematics. Given some initial/ boundary value problem, an integral equation is set up whose solution can be used to generate the solution of the parent problem. Unfortunately there are examples (Ursel1 [9], Jaswon and Symm [5]) where an integral equation may fail to have a solution even though there is no difficulty with the underlying boundary value problem. It is therefore desirable to have available an existence theorem, particularly when an exact solution cannot be determined so that asymptotic or numerical techniques are needed.

In this note a class of problems is considered which can be reduced to Fredholm integral equations of the first kind. The kernels of the integral equations are weakly singular with a logarithmic singularity; the integral equations arise in exterior boundary value problems in which Oseen's approximation (described in section 2) is

Received 18 August 1983

Copyright Clearance Centre, Inc. Serial-fee code: 0004-9727/84 $\$ A 2.00+0.00$ 
used. It is shown that provided the boundary value problem has no more than one solution, the corresponding integral equation always has a solution (within some suitable Hilbert space, for a class of prescribed functions which includes the physically reasonable cases; existence of solutions of the boundary value problem then follows).

The results are proved by expressing the integral operator as the sum of a singular part plus the regular part. An explicit representation of the inverse operator for the singular part is used to obtain an equivalent Fredholm equation of the second kind. The new integral operator is shown to be compact and the Fredholm alternative theorem, combined with the uniqueness theorem for the boundary value problem are used to deduce the existence of solutions of the original integral equation.

\section{Oseen type integral equations and some related boundary value problems}

There are many problems in viscous flow, heat transfer, chemical reaction theory and so on which can be classified as steady diffusionconvection problems. A field quantity such as heat or vorticity is produced at internal surfaces and is distributed around the region exterior to the boundaries by the processes of diffusion and forced convection. Certain conservation principles may be used to construct a system of partial differential equations satisfied by the field quantities. Often the field quantity of primary interest affects the externally driven flow causing a coupling of the partial differential equations; the coupling may occur non-linearly rendering the equations intractable in many important situations (non-linearities can also arise through the influence of field quantities on the diffusion coefficients, but these are not considered here). An important linearisation known in viscous flow as Oseen's approximation [8], can be applied to problems in which the forced flow field is unidirectional with constant velocity. In this approximation the perturbation to the external flow field is ignored in certain of the convection terms; in two-dimensional heat problems one obtains (see [1])

$$
\operatorname{div}(-\kappa \underline{\nabla T}+\underline{q T})=0, \underline{q}=U \hat{i}
$$


which imply that the temperature $T$ satisfies

$$
-\nabla^{2} T+\frac{U}{\kappa} \frac{\partial T}{\partial x}=0
$$

while in two-dimensional viscous flow the vorticity $\omega$ and stream function $\psi$ satisfy

$$
\operatorname{div}(-v \underline{\nabla} \omega+q \omega)=0,-\left(\nabla^{2} \psi\right) \underline{\hat{k}}=\omega \underline{\hat{k}}, \underline{q}=\operatorname{curl}(\psi \underline{\hat{k}})
$$

so with $\underline{q}=U \hat{i}$ in (2.3a) the stream function satisfies

$$
\nabla^{2}\left(\nabla^{2} \psi-\frac{U}{v} \frac{\partial \psi}{\partial x}\right)=0 .
$$

$(\kappa, \nu$ and $U$ are respectively, thermal diffusivity, viscosity, and free stream velocity.)

Problems which involve a flat plate set tangential to a uniform stream are particularly appropriate to this approximation. Green's functions which take account of the far field boundary conditions may be determined for these problems enabling the temperature field and velocity fields corresponding to equations $(2.2)$ and $(2.4)$ to be represented as follows,

$$
T(x, y)=\int_{-1}^{1} f(t) e^{\lambda(x-t)} K_{0}\left(\lambda\left[(x-t)^{2}+y^{2}\right]^{\frac{1}{2}}\right) d t
$$

and

(2.6) $u-i v=\frac{\partial \psi}{\partial y}+i \frac{\partial \psi}{\partial x}=\int_{-1}^{1} f(t)\left[\lambda e^{\lambda(x-t)}\left\{K_{0}(\lambda r)+K_{1}(\lambda r) e^{-i \theta}\right\}-\frac{e^{-i \theta}}{r}\right] d t$, where $r \cos \theta=x-t$, and $r \sin \theta=y$.

[The coordinates have been scaled so that the flat plate, of length $2 l$ in the unscaled system, is placed at $y=0,-1 \leqq x \leqq 1$. The constant $\lambda$ is a non-dimensional quantity given by

$$
\lambda=\frac{U \ell}{\kappa} \text {, or } \frac{U \ell}{v}
$$

which is commonly called the Peclet number in heat transfer or Reynold's number in viscous flow; it characterises the ratio of the convective flux to diffusive flux, of heat, or circulation.]

Applying the boundary conditions on $[-1,1]$, (prescribed temperature or velocity) we obtain the following well known integral equations, 


$$
\int_{-1}^{1} f(t) e^{\lambda(x-t)} K_{0}(\lambda|x-t|) d t=g(x)
$$

and

$$
\int_{-1}^{1} f(t)\left[\lambda e^{x-t}\left(K_{0}(\lambda|x-t|)+\frac{|x-t|}{(x-t)} K_{1}(\lambda|x-t|)\right)-\frac{1}{x-t}\right] d t=g(x) .
$$

Further problems in which the Oseen approximation has been used have been discussed recently ([4] and [7]) with a derivation of the concomitant integral equations given in each case. Both integral equations are Fredholm first kind with difference kernels which have a logarithmic singularity and decay to zero for large values of their arguments. For convenience these will be referred to as 'Oseen type' integral equations.

Fredholm equations of the first kind on a finite interval are difficult to solve and it appears unlikely that closed form solutions of equations (2.7) or (2.8) can be found. Recourse must be made to numerical and asymptotic techniques therefore (see [6]). Before embarking on such procedures an existence theory is desirable. There are circumstances in which integral equations derived from exterior boundary value problems fail to have solutions, although the boundary value problems do have solutions. This is true of the first kind equation on $(-2,2)$ having $\log |x-t|$ as its kernel (see [5]). Similarly integral equations which arise from exterior problems in acoustics (see [9]) also fail to have solutions. In the following sections it is shown that integral equations of Oseen type do not suffer from this deficiency.

\section{Existence of solutions of Oseen type integral equations}

It will now be shown that integral equations of Oseen type always have a unique solution provided the corresponding exterior boundary value problem has no more than one solution.

The essence of the proof is to express the kernel as the sum of a logarithmic part plus a remainder whose derivative is square integrable. 
The inverse operator for the logarithmic part is applied to obtain an equivalent second kind equation. Once the operator in the second kind equation has been shown to be compact the conclusions of the Fredholm alternative theorem together with the uniqueness properties of the exterior boundary value problem ensure the existence of a unique solution of the original equation.

These steps can be carried through only in the correct function space setting. In addition a suitable representation of the inverse operator for the logarithmic part of the kernel is needed. The definitions and representation given here can be found in Wolfe [10] in the context of an integral equation arising from solutions of the reduced wave equation. Define

$$
L_{2(p)} \equiv\left\{f: \int_{-1}^{1}|f(t)|^{2}\left(1-t^{2}\right)^{-\frac{1}{2}} d t \text { exists }\right\}
$$

and

$$
W_{2(q)}^{1} \equiv\left\{f: f \text { is absolutely continuous and } \int_{-1}^{1}\left|f^{\prime}(t)\right|^{2}\left(1-t^{2}\right)^{\frac{1}{2}} d t \text { exists }\right\}
$$

Under the norms

$$
\|f\|_{L_{2(p)}}^{2}=\int_{-1}^{1}|f(t)|^{2}\left(1-t^{2}\right)^{-\frac{1}{2}} d t
$$

and

$$
\|f\|_{W_{2(q)}^{1}}^{2}=\int_{-1}^{1}|f(t)|^{2}\left(1-t^{2}\right)^{\frac{1}{2}} d t+\int_{-1}^{1}\left|f^{\prime}(t)\right|^{2}\left(1-t^{2}\right)^{\frac{1}{2}} d t
$$

these are (complete) Hilbert spaces. On $L_{2(p)}$ the inner product is defined by

$$
\left(\phi_{1}, \phi_{2}\right)_{2(p)}=\int_{-1}^{1} \frac{\phi_{1}(t) \overline{\phi_{2}(t)}}{\sqrt{1-t^{2}}} d t .
$$


Integral equations of Oseen type have difference kernels $k(x-t)$ which are expressible as

$$
k(u)=\operatorname{cog}|u|+\sum_{n=0}^{\infty} a_{n} u^{n}+\log |u| \sum_{n=1}^{\infty} b_{n} u^{n}=k_{S}(u)+k_{R}(u),
$$

$$
\text { with } k_{S}(u)=\operatorname{clog}|u| \text {, }
$$

where the sums are analytic for all $|u| \geq 0$. The integral operators $K, K_{S}$ and $K_{R}$ are defined by

$$
\text { (3.7) } K f(x) \equiv \int_{-1}^{1} \frac{f(t)}{\left(1-t^{2}\right)^{\frac{1}{2}}} k(x-t) d t, K_{S} f(x) \equiv \int_{-1}^{1} \frac{c f(t)}{\left(1-t^{2}\right)^{\frac{1}{2}}} \log |x-t| d t \text { and } K_{R}=K-K_{S^{*}}
$$

The operator $K_{S}$ maps $L_{2(p)}$ onto $w_{2(q)}^{1}$, the latter space being characterised by the fact that (wolfe, [10]) $g$ belongs to $w_{2(q)}^{1}$ if and only if

$$
\sum_{n=0}^{\infty} n^{2}\left|\left(g, T_{n}\right) L_{2(p)}\right|^{2}<\infty
$$

$T_{n}$ is the Chebyshev polynomial of degree $n ;$ Chebyshev polynomials are orthogonal on $L_{2(p)}$. In view of the identities

$$
\frac{1}{\pi} \int_{-1}^{1} T_{n}(t)\left(1-t^{2}\right)^{-\frac{1}{2}} \log (|x-t|) d t= \begin{cases}-\log 2, & n=0, \\ -\frac{T_{n}(x)}{n}, & n \geqq 1,\end{cases}
$$

it follows from $(3.8)$ that $K_{S}^{-1}$ maps $w_{2(q)}^{1}$ onto $L_{2(p)}$; it is defined explicitly by

$$
K_{S}^{-1} g \equiv \frac{-\left(g, T_{0}\right) T_{0}}{c \pi \log 2}+\sum_{n=1}^{\infty}\left(\frac{-n}{c \pi}\right)\left(g, T_{n}\right) T_{n}
$$

[There are closed form expressions for $K_{S}^{-1}$, see carrier et al [2], for example, but they are too cumbersome to permit the analysis given here.] 
Let $q$ belong to $W_{2(q)}^{1}$ and denote an integral equation of Oseen type such as $(2.7)$ or $(2.8)$ by

$$
K f=g \text {. }
$$

Express the equation as

$$
\left(K_{S}+K_{R}\right) f=g
$$

and apply $K_{S}^{-1}$ to obtain

$$
f+K_{S}^{-1} K_{R} f=K_{S}^{-1} g
$$

Now $k_{R}^{\prime}(u)$ has a logarithmic singularity at $u=0$ and is otherwise analytic. This is enough to ensure that $K_{R}$ maps $L_{2(p)}$ into $w_{2(q)}^{1}$; thus for any $g$ in $W_{2(q)}^{I}$, equation (3.13) is an equation from $L_{2(p)}$ to $L_{2(p)}$ and is equivalent to (3.11).

Now it will be shown that $K_{S}^{-1} K_{R}$ is a compact operator from $L_{2(p)}$ to itself. Note that

$$
\text { (3.14) } \quad K_{S}^{-1}=\lim _{n \rightarrow \infty} K_{S n}^{-1}, K_{S n}^{-1} \equiv(-\pi \log 2)^{-1}\left(\cdot, T_{0}\right) T_{0}+\sum_{i=1}^{n}\left(\frac{-i}{\pi}\right)\left(\cdot, T_{i}\right) T_{i}
$$

where a pointwise limit is meant in (3.14). It will be shown that

$$
K_{n S}^{-1} K_{R} \rightarrow K_{S}^{-1} K_{R}
$$

in the $L_{2(p)}$ norm, from which the compactness of $K_{S}^{-1} K_{R}$ follows, since each $K_{n S}^{-1} K_{R}$ is of finite rank, and therefore compact.

Denote by $\sigma_{m n}$ the expression

$$
\sigma_{m n}=\left\|K_{S n}^{-1} K_{R} f-K_{S m}^{-1} K_{R} f\right\|_{L_{2(p)}}^{2}
$$


Suppressing the $L_{2(p)}$ suffix, we have

(3.17) $\pi^{2} \sigma_{m n}=\left\|\sum_{i=m+1}^{n} i\left(K_{R} f, T_{i}\right) T_{i}\right\|^{2}=\sum_{m+1}^{n}\left|i\left(K_{R} f, T_{i}\right)\right|^{2}=\sum_{m+1}^{n}\left|\left(f, i K_{R}^{*} T_{i}\right)\right|^{2}$.

The properties of the Chebyshev polynomials enable $K_{R}^{*} T_{i}$ to be integrated by parts so that

$$
K_{R}^{*} T_{i}=\frac{1}{2 i}\left(\left(K_{R}^{*}\right) T_{i-1}-\left(K_{R}^{*}\right) T_{i+1}\right) \text {, where } K_{R}^{*} f \equiv \int_{-1}^{1} \frac{f(t)}{\left(1-t^{2}\right)^{\frac{1}{2}}}\left(\frac{\partial}{\partial t} k(t-x)\right) d t
$$

Thus applying this to (3.17) and applying the Cauchy-Schwarz inequality we have

$$
\pi^{2} \sigma_{m n} \leq \frac{1}{4}\|f\|^{2}\left(\sum_{m+1}^{n}\left(\left\|K_{R}^{*}{ }^{\prime} T_{i-1}\right\|^{2}+\left\|K_{R}^{*}{ }^{\prime} T_{i+1}\right\|^{2}\right)\right)
$$

Now if the $K_{R}^{*}$, operator is written out explicitly, in view of (3.6) it is easily observed that $K_{R}^{*}$ ' can be similarly expressed; that is,

$$
K_{R}^{*}=c_{1} K_{S}+K_{T}
$$

and $K_{S}$ is the same operator as defined at (3.7). Thus

$$
\left(K_{R}^{*}{ }^{\prime}\right) T_{i}=c_{1} K_{S} T_{i}+K_{T} T_{i}
$$

and so applying the relations (3.9) to the first term and the equation (3.18) to the second term on the right hand side of (3.21) the estimates

$$
\left\|K_{R}^{*} T_{i \pm 1}\right\|^{2} \leqq \frac{c_{1}^{2} \pi^{3}}{i^{2}}+\frac{\pi}{2 i^{2}}\left\|\left(K_{T}^{* \prime}\right)\right\|^{2}
$$

are obtained. These ensure that for any preassigned $\varepsilon$, and $m$ and $n$ large enough,

$$
\sigma_{m n} \leqq\|f\|^{2} \varepsilon
$$

and the validity of $(3.15)$ in the $L_{2}(p)$ norm is demonstrated. 
Now the Fredholm alternative may be applied to (3.13). In the event of (3.13) having a solution for zero right hand side, then (3.11) would have the same solution. This would generate a non-trivial solution to the exterior boundary value problem for prescribed data equal to zero. Hence, provided the boundary value problem has suitable uniqueness properties which can be deduced independently of the arguments presented here, equation (3.13) could not have a non-trivial solution for zero data, and thus for any $K_{S}^{-1} g$ in $L_{2(p)}$, i.e. any $g$ in $W_{2(q)}^{1}$, a unique solution of (3.13), and therefore (3.11) also, exists.

\section{Concluding remarks}

The existence of solutions of equations (2.7) and (2.8), for any $g$ in $w_{2(q)}^{1}$, follows from the results of section 3 , since the corresponding heat transfer and viscous flow problems can be shown to have at the most one solution. In the temperature problem a maximum principle can be proved for the operator $\left(\nabla^{2}-\lambda^{2}\right)$ which is obtained after removal of the first derivative term by expressing the solutions in terms of $e^{-\lambda x} T(x, y)$. In both problems energy arguments can also be given (see Finn [3] for the viscous problem). In the viscosity problem the uniqueness results strictly apply to the perturbation in the velocity field from the free stream since any solution for $\psi$ is only determined up to an additive constant.

The proof of existence of solutions of equation (2.7) could be simplified somewhat by combining the exponential terms in the kernel with $f$ and $g$. In this new form the kernel has an expansion with the term $u \log |u|$ (indeed all terms $u^{2 n+1} \log |u|$ ) absent; this allows two integration by parts, whereas only one was used to obtain (3.19), and thereby shortens the argument leading to (3.23).

A key factor in proving these results is the decay rate at infinity of the field variables. Note that the corresponding potential problems do not have these properties; this perhaps explains the non-existence for the logarithmic kernel on $[-2,2]$.

As far as the examples from [4] and [7] are concerned, it is conjectured that uniqueness theorems for the exterior boundary value problems can be proved but this has yet to be attempted. 


\section{References}

[1] J.A. Belward, "Forced convection problems and some related Fredholm integral equations of the first kind", (Ph.D. thesis, University of Queensland, 1971).

[2] G.F. Carrier, M. Krook and C.E. Pearson, Functions of a complex variable, theory and technique, (McGraw Hill, New York, 1966).

[3] R. Finn, "On the exterior stationary problem for the Navier-Stokes equations and associated perturbation problems", Arch. Rat. Mech. Anal., 19 (1965), 363-406.

[4] D. Homentcovschi, "Oseen flow of a compressible fluid past a flat plate", Quart. App 2. Math. 39 (1981), 221-237.

[5] J.A. Jaswon and G.T. Symm, Integral equation methods in potential theory and elastostatics, (Academic Press, London, 1977).

[6] T. Miyagi and M. Nishioka, "Oseen velocity distributions in the wake of a flat plate", J. Eluid. Mech. 97 (1980), 145-155.

[7] W.E. Olmstead and S.R. Majumdar, "Fundamental Oseen solutions for the two-dimension flow of a micropolar fluid", Int. J. Engng. Sc. 21 (1983), 423-433.

[8] L. Rosenhead, Laminar boundary Zayers, (clarendon Press, Oxford, 1963).

[9] F. Ursel1, "On the exterior problems of acoustics", Math. Proc. Comb. Phil. Soc. 74 (1973), 117-125.

[10] P. Wolfe, "Some distribution solutions of integral equations and their application to partial differential equations", Applicable Analysis, 1 (1971), 123-141.

Department of Mathematics

University of Queensland

St. Lucia, Q1d. 4067

AUSTRALIA 